\title{
Astrocitoma intramedular grado III en paciente de 21 meses de edad: descripción de un caso
}

Publicado en Internet: 19-juniol-2015

Francisco Medrano Muñoz: pancho.medrano@live.com.mx

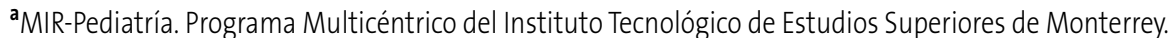
Secretaria de Salud del Estado de Nuevo León. México • Neurología Pediátrica. Centro Médico San José.

Nuevo León. México.
Palabras clave:

- Astrocitoma

intramedular

- Tumoración - Niño
Los tumores del sistema nervioso central (SNC) representan el $15-20 \%$ de todas las neoplasias en la población pediátrica. Los tumores del cordón espinal son raros en la niñez y constituyen solo el 4-10\% de todos los tumores primarios del SNC. Los tumores intramedulares son los menos comunes y constituyen alrededor del $35 \%$ de todos los tumores espinales en niños. Los astrocitomas son las neoplasias intramedulares más comunes presentándose hasta en un $75 \%$, de estos, solo el $25 \%$ aproximadamente son de grado III. Con el fin de enfatizar sobre las presentaciones clínicas y métodos diagnósticos de esta enfermedad, se describen las manifestaciones clínicas así como el método diagnóstico de un astrocitoma intramedular grado III en un paciente femenino de 21 meses de edad.

Los tumores espinales en niños presentan una amplia gama de signos y síntomas clínicos, razón por la cual pueden representar un reto para la sospecha y el diagnóstico de la localización intramedular. Se recomienda tener un alto índice de sospecha al presentar síntomas neurológicos. La resonancia magnética simple y contrastada es el estudio de imagen de elección para las tumoraciones intramedulares. El único método para su diagnóstico y estatificación es la biopsia.

\section{Intramedullary astrocytoma grade III in a 21-month-old patient: case report}

Central nervous system (CNS) tumors represent $15-20 \%$ of all tumors in childhood. Primary spinal cord tumors are a rare entity that make up to only $4-10 \%$ of all primary tumors of the CNS. Intramedullary tumors are the least common type of spinal tumors, only $35 \%$. The most common intramedullary tumor is the astrocytoma, $75 \%$ of the cases, and only in $25 \%$ are of grade III. In order to emphasize the clinical presentation as well as the diagnostic methods of this disease, we present the clinical manifestations and the diagnostic method of an intramedullary astrocytoma grade III in a 21-month-old patient. Spinal cord tumors in children present a wide variety of clinical signs and symptoms; this is why the Key words: $\quad$ suspicion and diagnostic of intramedullary tumors can be challenging to the physician. A high degree

- Intramedullary astrocytoma grade III of suspicion is advisable when a child presents neurological findings. The magnetic resonance imaging with and without contrast is the study of choice for all intramedullary tumors. The only method useful

- Tumor

- Child for the diagnosis and stadification is biopsy.

\section{INTRODUCCIÓN}

Los tumores del sistema nervioso central (SNC) representan el 15-20\% de todas las neoplasias en la población pediátrica. Los tumores del cordón espinal son raros en la niñez y constituyen solo el
4-10\% de todos los tumores primarios del $\mathrm{SNC}^{1}$, se reportan aproximadamente 10-30 casos en pacientes menores de tres años al año en Estados Unidos de Norte América². La clasificación macroscópica de los tumores de cordón espinal, se basa en la localización y se divide en dos tipos: a) tumores

Cómo citar este artículo: Medrano Muñoz F, Garza Peña A. Astrocitoma intramedular grado III en paciente de 21 meses de edad: descripción de un caso. Rev Pediatr Aten Primaria. 2015:17:e133-e136. 
extradurales, los cuales se localizan en el área entre las estructuras óseas y la duramadre, y b) tumores intradurales, los cuales se subdividen en extramedulares e intramedulares.

Los tumores intramedulares son los menos comunes y constituyen alrededor del 35\% de todos los tumores espinales en niños. Los astrocitomas son la neoplasia intramedular más común, presentándose hasta en un $75 \%$, de estos, solo el $25 \%$ aproximadamente son grado $\mathrm{II}^{1}$, según la clasificación de la Organización Mundial de la Salud (OMS).

Las localizaciones primarias del tumor espinal intramedular más común, según un estudio retrospectivo realizado de Constantini et al., en pacientes menores de tres años es: $18,5 \%$ cervical, 33\% cervicotorácica y $48,1 \%$ torácica ${ }^{2}$.

Sin embargo, la edad más común de presentación en la edad pediátrica es en pacientes mayores de cinco años, pero en un estudio retrospectivo realizado en un centro de alta concentración para pacientes con tumoraciones en canal medular en Canadá, donde se incluyeron todos los niños menores de tres años con una tumoración en la médula espinal, realizado por Zeleer et al., se demuestra que la edad de presentación más común en este grupo de edad es entre los 13 y los 24 meses $^{3}$. El alto índice de sospecha es de vital importancia para realizar el diagnostico de cualquier tumoración en la médula espinal, dado que presentan una amplia gama de signos y síntomas clínicos, los cuales pueden variar de ser muy sutiles hasta ser muy llamativos, regularmente relacionándose a un peor pronóstico según el tamaño y localización dentro de la médula espinal. En el estudio realizado por Zeleer et al. en esta población pediátrica, los síntomas iniciales de las tumoraciones intramedulares son los siguientes: alteraciones en la marcha en un $72,7 \%$, regresión del desarrollo en un $27,2 \%$, movimientos anormales del ojo en el 9,1\%, convulsiones en el 4,5\%, lateralización de la cabeza en el 4,5\%, vómitos recurrentes en el $4,5 \%$, fallo de medro en el $4,5 \%$, aumento del perímetro cefálico y dolor en el $0 \%^{3}$.

En el estudio realizado por Constantini et al. se mencionan los principales síntomas que observaron los padres de niños menores de tres años de edad que presentaron una tumoración intramedular antes del diagnóstico: dolor en el $42 \%$, regresión motora en el $36 \%$, alteraciones a la marcha en el $27 \%$, torticolis en el $27 \%$, escoliosis en el $24 \%$, retraso en el desarrollo psicomotor en el $12 \%$, retención urinaria en el $8 \%$ y otros en el $4 \%^{2}$.

Existe una escala utilizada exclusivamente para la evaluación funcional, según la clínica presentada en los pacientes con una tumoración intramedular, llamada McCormick modificada 4 .

Una vez realizado el diagnóstico de neoplasia intramedular por medio de imagen es necesario realizar una biopsia, y la frecuencia de los tipos histológicos encontrados dentro del estudio realizado por McGirth et al. fue la siguiente ${ }^{1}$ : astrocitomas en un $75 \%$ (grado I en un $25 \%$, grado II en un $33,3 \%$, grado III en un $25 \%$ y grado IV en un $16 \%$ ), gangliomas en un $12,5 \%$, ependimomas en un 6,5\% y gliomas en un 6,5\%.

\section{CASO CLÍNICO}

Paciente del sexo femenino, sin antecedentes de importancia, de 21 meses de edad. Refiere la madre que inicia su padecimiento actual 11 días previos a su ingreso, al detectar de manera súbita alteración de la marcha, descrito como "arrastrando el pie izquierdo"; esto no le causaba pérdida del equilibrio, caída o inhabilidad para desplazarse. Así mismo, nota debilidad del brazo ipsilateral con disminución de los movimientos y discapacidad para manipular objetos, caracterizada por pasarlos a la otra mano, niega el poder localizar con exactitud si es de predominio proximal o distal; esto se presenta de manera constante, sin ceder, sin horario o calendario, sin atenuantes, sin exacerbantes o acompañantes.

A la exploración neurológica se encuentran funciones superiores preservadas, nervios craneales sin alteraciones, sistema sensorial -lo posible de valorar por la edad y cooperación del paciente-normal, a la inspección muscular se encuentran las cuatro extremidades simétricas y eutróficas con tono muscular sin alteraciones, la fase activa de las 
extremidades se encontró fuerza $3 / 5$ en porcion distal y $4 / 5$ en seccion proximal de hemicuerpo izquierdo y $5 / 5$ en extremidades de hemicuerpo derecho. No presenta dificultad para mantener la bipedestación sin apoyo. La marcha se observa sin aumento de la base de sustentación, con una fase de contacto simetrica bilateral, pero con discreta rotación externa de todo el miembro pelvico izquierdo con caída del pie golpeando el suelo con toda la planta y sin aumento de la fase de oscilación, con movimientos asimétricos, acompañados de extensión incompleta de la rodilla izquierda. Puede realizar la marcha sin apoyo. Reflejos primitivos ausentes, Babinski negativo, reflejo braquioradial $2 / 4$, bicipital $2 / 4$, tricipital $2 / 4$, rotulaino 2/4, aquileo 2/4 bilaterales. Rigidez nucal, signo de Brudsinski y signo de Kernig negativos.

Se solicitan radiografías de columna y cadera, que se reportan como normales y se le solicita una tomografía computarizada (TC) simple de cráneo, sin encontrar datos radiográficos sugestivos del origen de la causa de la sintomatología, razón por la cual se le solicita una resonancia magnética (RM) simple y contrastada de cráneo y médula espinal, encontrándose una lesión intramedular heterogénea que se extiende desde C 3 hasta T3 con zonas hipointensas en T1 y zonas hiperintensas en T2 que involucran el espesor del canal medular y la cual mide 7,6 cm de longitud y oblitera el espacio subaracnoideo (Fig. 1).

Se preparó a la paciente y se realizó biopsia, extrayéndose muestras para análisis por parte del Servicio de Patología, que observó neoplasia fibrilar compuesta de hipercelularidad de tipo astrocítica con áreas de hemorragia; no se observó necrosis ni hiperplasia vascular, se encontraron células irregulares, cromatina grumosa y no se observó ninguna mitosis, realizándose el diagnostico de astrocitoma fibrilar difuso grado II de la OMS de localización intramedular (Figs. 2 y 3 ).

Posteriormente se traslada la paciente a un centro especializado en la patología en los EE. UU., donde se realiza la extracción de la mayor cantidad de tumoración, aproximadamente un $80-90 \%$, y se envía la muestra a patología donde se encuentran dos mitosis positivas, reclasificando la tumoración en un grado III de la OMS.

Figura 1. Resonancia magnética simple y contrastada de cráneo y medula espinal. Se encuentra una lesión intramedular heterogénea que se extiende desde C 3 hasta T3 con zonas hipointensas en T1 y zonas hiperintensas en T2 que involucran el espesor del canal medular. La lesión mide $7,6 \mathrm{~cm}$ de longitud y oblitera el espacio subaracnoideo
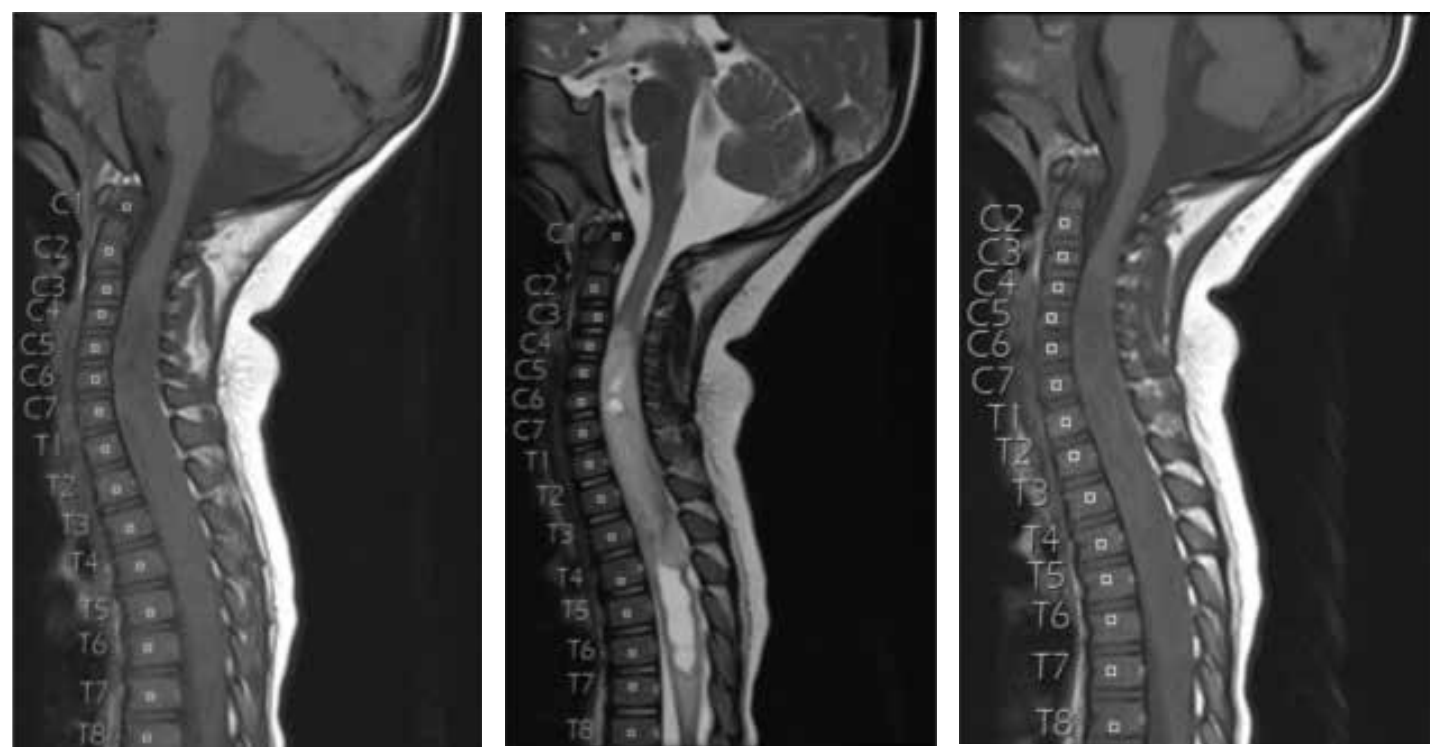


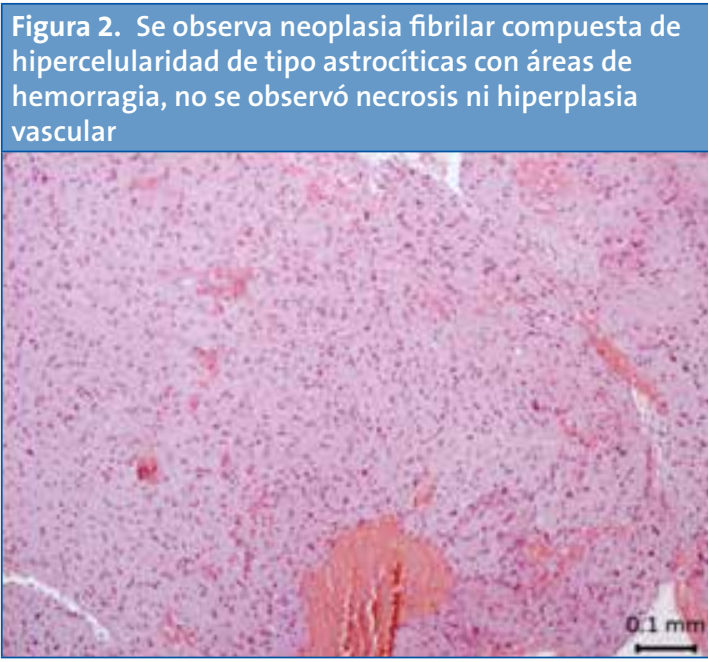

\section{DISCUSIÓN}

Por lo antes mencionado, los astrocitomas primarios intramedulares en la población pediátrica son una patología poco común, que puede pasar desapercibida por un tiempo indefinido.

Según la clasificación de McCormick modificada, nuestra paciente se puede clasificar en un grado II, dado el grado de incapacidad que presenta al momento de realizar el diagnóstico.

Es imperativo el alto índice de sospecha para poder llegar a un diagnóstico, sin embargo, en la actualidad no existe una clasificación realizada intencionadamente para la población pediátrica; la clasificación

\section{BIBLIOGRAFÍA}

1. McGirt M, Chaichana K, Atiba A, Attenello F, Woodworth G, Jallo G. Neurological outcome after resection of intramedullary spinal cord tumors in children. Childs Nerv Syst. 2008;24:93-7.

2. Constantini S, Houten J, Miller D, Freed D, Ozek M, Rorke L, et al. Intramedullary spinal cord tumors in children under the age of 3 years. J Neurosurg. 1996; 85:1036-43.
Figura 3. A mayor aumento encontramos células irregulares, cromatina grumosa, y no se observa ninguna mitosis

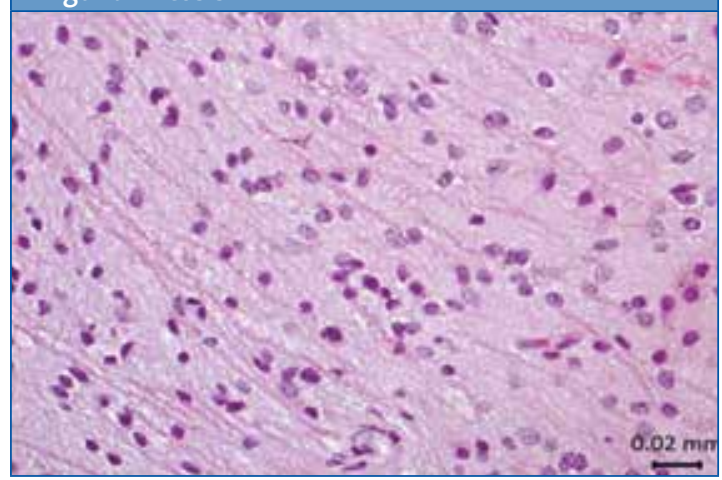

que actualmente existe y la más ampliamente usada es la de la Organización Mundial de la Salud (OMS), que se realizó con estudios en la población adulta, así que es necesario un mayor estudio de la presentación, tratamiento y pronóstico de esta patología en la edad pediátrica.

\section{CONFLICTO DE INTERESES}

Los autores declaran no presentar conflictos de intereses en relación con la preparación y publicación de este artículo.

\section{ABREVIATURAS}

OMS: Organización Mundial de la Salud • RM: resonancia magnética • SNC: sistema nervioso central • TC: tomografía computarizada.

3. Zeleer S, Jeene D, Bartels U, Carret A, Crooks B, Eisentat D, et al. Spinal cord tumors in children under the age of 3 years: A retrospective Canadian review. Childs Nerv Syst. 2011;27:1089-94.

4. Herkowitz H, Garfin S. Eismont F, Bell G, Balderston R. Intradural tumor. En: Rothman S. The Spine Expert Consult. 6. ${ }^{\text {a }}$ edición. Londres: Saunders; 2011. p. 1612-7. 